\title{
A New Weighed Recursive Filter Constant False Alarm Rate Detection Algorithm in Multiple-target Interference Process
}

\author{
Chaoyi Luo, Siyi Chen, Yubing Wang \\ Aeronautical and Astronautical Engineering College, the Air Force Engineering University, Xi'an 710038, \\ Shanxi, China
}

\begin{abstract}
For multi-target interference reference unit in the process of target detection unit, presenting a new CFAR algorithm. The algorithm based on the history of the selected reference samples respectively weighted recursive estimate clutter power level. Simulation results show that weighted recursive filter in eliminating interference and target detection in clutter edge showed good performance, WRF-CFAR is more desirable in the fight against clutter edge. The overall performance by the improved CWRF-CFAR was better than WRF-CFAR and with the increase of the length of filtering the lead in the homogeneous clutter background.
\end{abstract}

KEYWORD: multi-target interference; constant false alarm rate; weighed recursive filter; clutter edge

\section{INTRODUCTION}

CFAR technique of signal has been widely used in radar system to accomplish signal detection, which is called constant false alarm processing (NI Fei, 2000). In the process of constant false alarm rate detection on multiple targets, other targets in the reference unit affect calculating decision threshold which would interfere with the target detection in detection unit. Different CFAR detection algorithms have different characteristics and applicable environment, thus, no detector can be applied to all cases (YUAN Chang, 2012). In the background of uniform clutter, CA-CFAR performs best in detection and is simplest. To build the detection covariance matrix of background noise according to characteristics of external noise so as to change threshold value adaptively under the multi-target environment or non uniform noise environment becomes an urging issue (GUI Ren-zhou, 2012). In addition to deal with multi-target environment is often put forward some improved CFAR algorithm such as improved SOCFAR and OS-CFAR(C.Yi, 2014).Some domestic universities and academic institutions are also put forward some algorithms such as broadband acoustic vector array target beam domain transformation of generalized likelihood ratio detection algorithm in reference Sampling for receiving data in different frequency sub-band beam domain by designing the beam domain transformation matrix. Reference realized SAR ship target CFAR detection based on the reflection symmetry polarization and Reference has done research on Statistical MIMO radar CFAR de- tector based on the technology of channel selection which proposed channel selection technology based on the order statistic test methods. The paper propose an algorithm using weighted recursive filter constant false alarm rate (WRF-CFAR). The algorithm can set filter threshold to control false alarm rate according to specific clutter environment and delete interference target sample adaptively without damaging sample data, thereby improving the detection performance.

\section{WEIGHTED RECURSIVE FILTER}

\subsection{Weighted Recursive Filter principle}

Weighted recursive algorithm aims at real time tracking of characteristic changes of the clutter, 'learning' according to clutter environment and adjust filtering threshold factor so as to filter out multiple targets. Optimal filter can be realized through using filter parameters formerly obtained to adjust parameters at present automatically in order to adapt to statistic characteristics of sample and target jamming. Weighted recursive algorithm for estimating the mean value is biased. The unbiased estimate is obtained by using a simple logic circuit. The flowchart of process above is shown in Fig.1.

Front and back down sliding window sample data can be obtained after demodulation of the received signal. Prediction threshold in flowchart can be obtained by using eq.(1). 


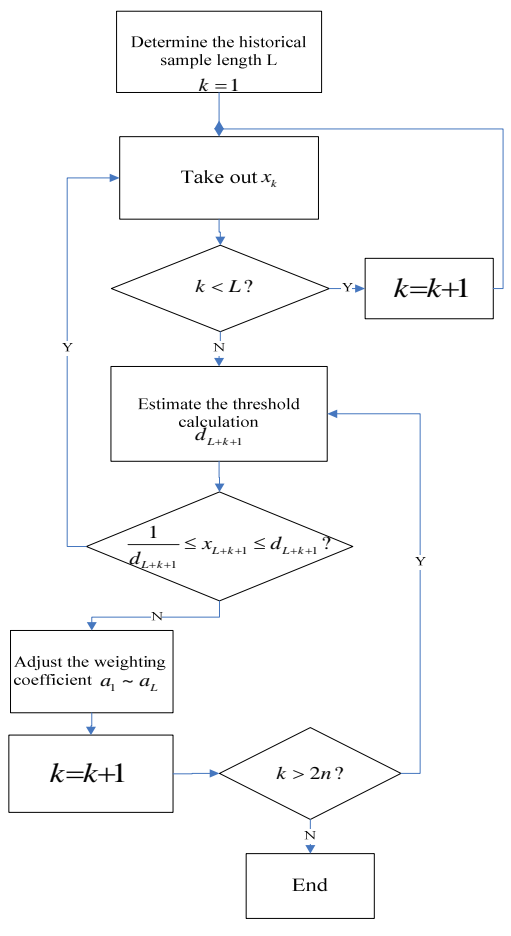

Fig.1 Weighed recursive filter flow chat

$$
\left[\begin{array}{c}
d_{1} \\
d_{2} \\
\vdots \\
d_{2 n-N+1}
\end{array}\right]=\left[\begin{array}{c}
\sum_{m=1}^{N} a_{m 1} x_{m} \\
\sum_{m=1}^{N} a_{m 2} x_{m+1} \\
\vdots \\
\sum_{m=1}^{N} a_{m(2 n-N+1)} X_{m+2 n-N}
\end{array}\right]
$$

When the value of sample exceeds the prediction threshold

$$
\left[\frac{1}{d_{i}}, d_{i}\right] \quad(1 \leq i \leq 2 n-N+1) \text {, abandon } x_{i+1} \text {, the }
$$

rest data builds up reference unit historical sample data, participate in the next round of computing.

\subsection{Weighed Recursive Filter Constant False Alarm Rate Processing}

A WRF-CFAR block diagram is shown in Fig.2. Assuming that front sliding window after filtering the remaining data in the array $\mathrm{X}$ along the sliding window in the $\mathrm{Y}$ array, and getting verdict results through comparator by calculating threshold value and testing units value.

Assuming that false alarm rate is $P_{f a}=10^{-6}$, theoretical threshold is expressed in $\mathrm{dB}[5]$, which is $K=20 \lg \sqrt{2 \ln \left(1 / P_{f a}\right)}=14.2 d B$

In uniform clutter background, the mean value of all the frontier and back sliding window data is $a=\frac{1}{2 n}\left(\sum_{i=1}^{2 n} x_{i}\right)$,assuming that filtering threshold factor is $\varphi$,filtering threshold is $\varphi a$,then the dynamic adjustment of the level of clutter power estimation can be realized. According to reference, Reference to draw adaptive threshold of WRF-CFAR which is shown in chart1. $(\bar{X}, \bar{Y}$ are the corresponding array for frontier and back sliding window after filtering)

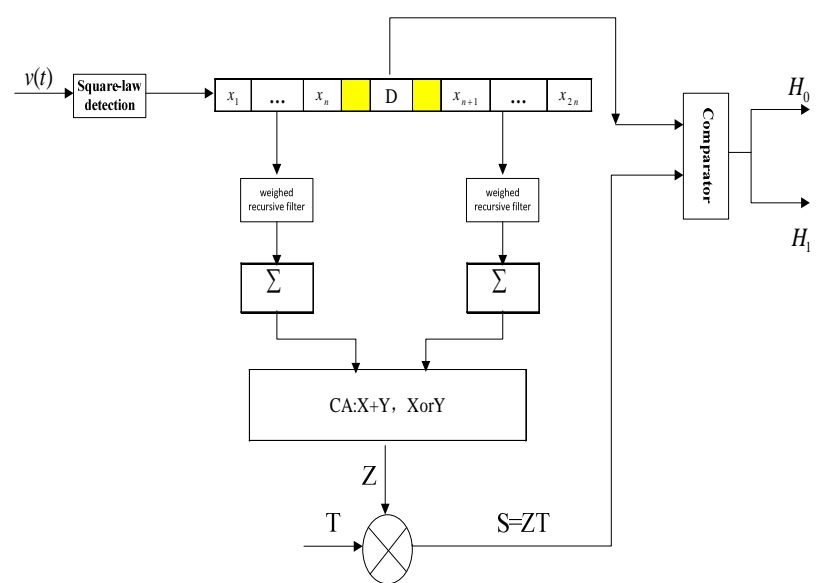

Fig.2 WRF-CFAR detector block diagram

Tab.1 WRF-CFAR adaptive threshold equivalent CFAR processing method

\begin{tabular}{lllll}
\hline $\begin{array}{l}\text { Serial } \\
\text { number }\end{array}$ & $\begin{array}{l}\text { Fliding } \\
\text { window } \\
\text { is } \\
\text { uniform }\end{array}$ & $\begin{array}{l}\text { Background } \\
\text { window } \\
\text { uniform }\end{array}$ & $\begin{array}{l}\text { The } \\
\text { mean } \\
\text { is the } \\
\text { same }\end{array}$ & $\begin{array}{l}\text { WRF- } \\
\text { CFAR } \\
\text { adaptive } \\
\text { threshold }\end{array}$ \\
\hline 1 & $\mathrm{~N}$ & $\mathrm{~N}$ & $\mathrm{Y}$ & $C_{2 n} \sum_{\bar{X} \bar{Y}}$ \\
2 & $\mathrm{Y}$ & $\mathrm{N}$ & - & $C_{n} \sum_{\bar{Y}}$ \\
3 & $\mathrm{~N}$ & $\mathrm{Y}$ & - & $C_{n} \sum_{\bar{X}}$ \\
\hline
\end{tabular}

Then, the clutter environment of target is judged by the multi scale statistical variance of the background window. Next, selecting the corresponding background clutter distribution model adaptively, and calculating corresponding prediction threshold. When two sliding windows are used, $C_{2 n}$ is adopted. When only one sliding window is used, $C_{n}$ is adopted.

\section{PERFORMANCE ANALYSIS OF WRF-CFAR IN MULTI TARGET ENVIRONMENT}

\subsection{Detection Analysis of WRF - CFAR in Uniform Clutter Background}

When background reverberation is distributed in Gaussian distribution, its envelope is Rayleigh distribution, the probability distribution is:

$$
f(x)=\frac{x}{\sigma^{2}} \exp \left(-\frac{x^{2}}{2 \sigma^{2}}\right) \quad \sigma>0, x \geq 0
$$

$\sigma$ is distribution parameter of Rayleigh distribution. 
In detector of WRF-CFAR, estimation of background clutter power level is acquired by calculating mean value of reference sampled $p+q$ units. In which $p$ represents the number of sampled values of the residual value of the frontier edge sliding window, $q$ represents the number of sampled values of the residual value of the back edge sliding window. The total clutter power level is estimated to be:

$$
Z=\sum_{i=0}^{p} x_{i}+\sum_{j=0}^{q} y_{j}
$$

Due to the uniform clutter background, the processing of data after filtering amounts to CA-CFAR.

It is difficult to obtain PDF closed type solution and IID random variable through square-law detector $^{[9]}$. The detection probability of single pulse WRF-CFAR detector is:

$$
P_{d}=\left(1+\frac{T}{1+\lambda}\right)^{p+q}
$$

$T$ represents nominal factor, $\lambda$ represents average power ratio of target signal and noise. The relationship between nominal factor and false-alarm probability is:

$T=\left(P_{f a}\right)^{-1 /(p+q)}-1$

when false-alarm probability values $P d=10^{-6}$.

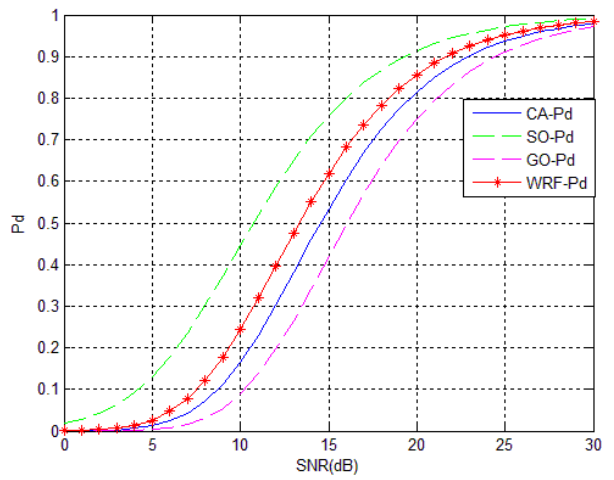

Fig.3 The compare of CA-CFAR with WRF-CFAR when $2 n=16$

Approximate expression of false-alarm probability for single pulse CA-CFAR detector is proposed in reference ${ }^{[10]}$ :

$P_{f a}=\left\{1+\frac{T^{2}}{(p+q)\left[c-(c-1) e^{-(p+q+1)}\right]}\right\}^{-(p+q)}$

In the formula $c=4 / \pi$, simulation results of WRF-CFAR with different length of sliding window are shown below:

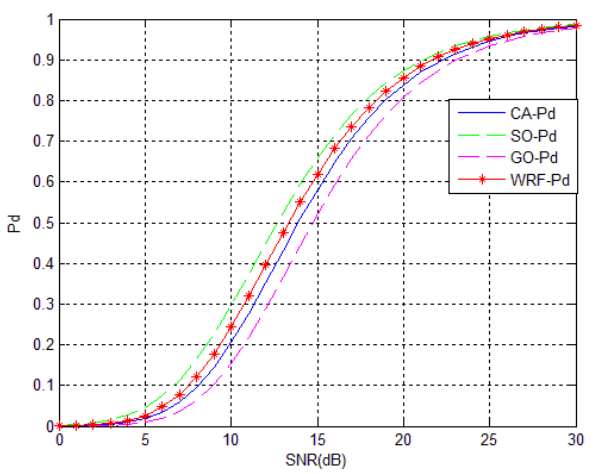

Fig.4 The compare of CA-CFAR with WRF-CFAR when $2 n=32$

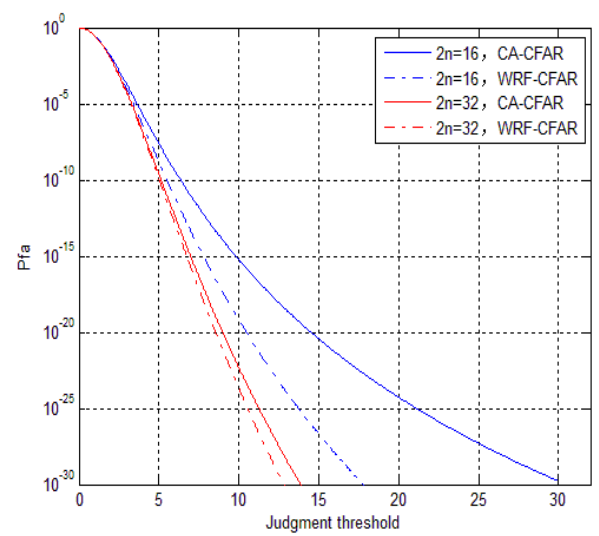

Fig. 5 The compare of CA-CFAR with WRF-CFAR when $2 \mathrm{n}$ is different

\subsection{Detection Analysis of WRF - CFAR in Clutter Edge Background}

When WRF-CFAR is in clutter edge environment, verifying the location of clutter edge is the first step, then judging clutter region which detection unit is in. the determine method is given in reference. For statistical independent clutter power level, its estimation is:

$Z=\sum_{i=1}^{N_{c}} x_{i}+\sum_{N_{c}+1}^{2 n} x_{i}=Z_{1}+Z_{2}$

Due to the statistical independence of $Z_{1}$ and $Z_{2}$, the moment generating function (MGF) of $Z$ is product of the respective MGF of $Z_{1}$ and $Z_{2}{ }^{[9]}$.The false alarm probability expression of the edge of the clutter located in frontier or back sliding window is given in reference and reference.

The location of changed clutter $N_{c} \leq n$, which means the clutter edge is in the frontier edge sliding window, the false alarm probability of WRF-CFAR is:

$P_{f a}=\frac{1}{(1+\gamma T)^{N_{c}}(1+T)^{2 n-N_{c}}}$ 
When $N_{c}>n$, which means the clutter edge is in the back edge sliding window, the false alarm probability of WRF-CFAR is:

$$
P_{f a}=\frac{1}{(1+T)^{N_{c}}(1+T / \gamma)^{2 n-N_{c}}}
$$

In the formula, $\gamma$ represents the ratio of two clutter power intensity.

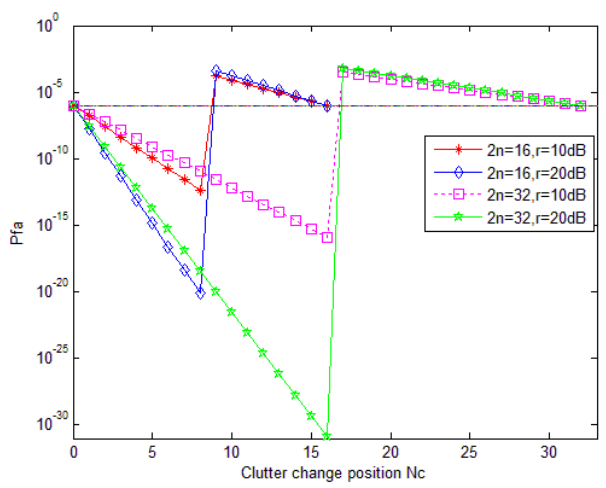

Fig.6 The WRF-CFA detector in suppressing clutter and noise

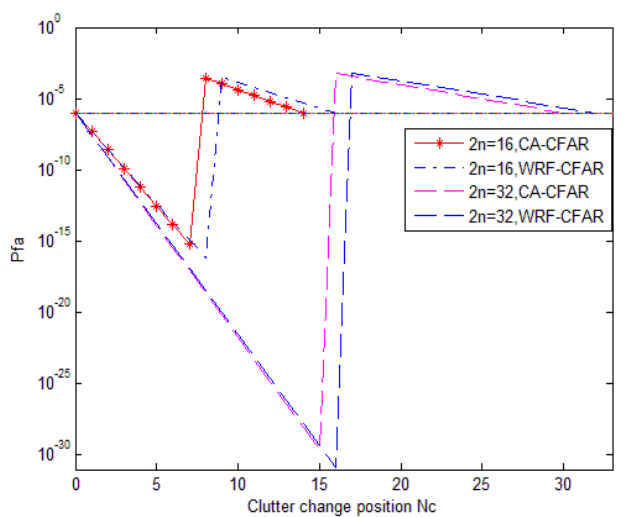

Fig.7 The compare of WRF-CFAR with CA-CFAR in suppressing clutter and noise

\section{DETERMINATION OF THE BEST HISTORICAL SAMPLE LENGTH}

The first step in WRF-CFAR process is to choose the historical length $\mathrm{N}$ of sampled. Because of the previous data $\mathrm{N}$ are not involved in calculating, leading to some data are wasted. If $\mathrm{N}$ is too small, result is not representative. However, with the increase of $\mathrm{N}$, other targets are covered and detection effect is weakened. Thus, a proper determination of $\mathrm{N}$ is important in improving the detection effect of WRF-CFAR.

Data utilization is signed as $1-l / 2 n$ and echo intensity coefficient is signed as $\mu$ ( $\mu$ is relevant with intensity of transmitted signal, distance, size of target, etc.), $m$ is proposed to represent the number of targets. Assuming that interference echo is evenly distributed in reference sliding window, the mathe- matical expectation and minimum mean square error of $m$ can be obtained:

$$
E(\mathbf{c})=\frac{1}{2 n-m+\left[\frac{m}{2 n}\right]}\left\{\sum_{i=1}^{2 n-m} \mathbf{c}[i]+\sum_{j=1}^{\left[\frac{m}{2 n}\right]} \lambda_{j}\right\} \quad(1 \leq l \leq 2 n)
$$

c'[i] represents the recombined array of sliding window after eliminating multiple targets. $E_{l}(\mathbf{c})$ represents the mathematical expectation of different historical reference sample. $\left[\frac{m l}{2 n}\right]$ is the integer conversion of $\frac{m l}{2 n}$. The corresponding variance is:

$e_{l}=\frac{1}{2 n}\left[\sum_{i=1}^{\left.2 n-\frac{m l}{2 n}\right]}\left|\mathbf{c}_{i}^{\prime}-E_{l}(\mathbf{c})\right|^{2}+\sum_{j=1}^{\left[\frac{m l}{2 n}\right]}\left|\lambda_{j}-E_{l}(\mathbf{c})\right|\right]$

Simulation result of variance is shown in Fig.8.

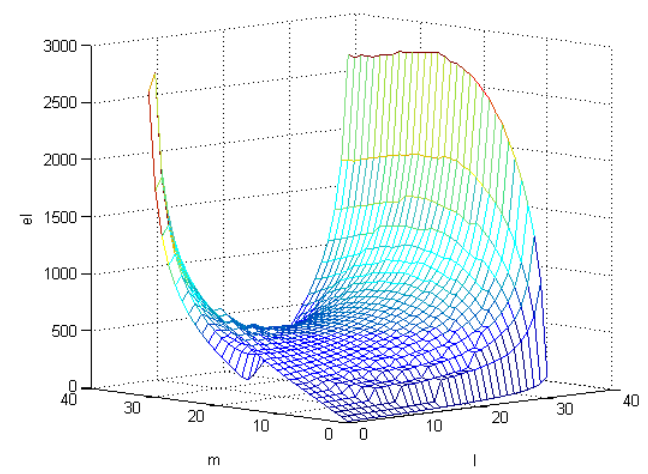

Fig.8 Corresponding variance to $\mathrm{m}$ and $\mathrm{l}$

The value of $m$ is determined by detection environment, after which the value of $l$ is selected corresponding to the lowest point of curve and the optimal filter length is determined.

\section{CYCLIC WEIGHTING RECURSIVE FILTERING CWRF-CFAR}

In the uniform clutter background, the decision threshold of CA-CFAR is obtained by averaging and normalizing all the data. When echo signal of multiple targets damages the uniform clutter background, WRF-CFAR can adjust threshold adaptively according to background noise and filter out the echo signal of jamming targets. Data after filtering is more close to uniform background; however, the reference units history are needed. Particularly, data utilization is not efficient enough when filter length is great. Thus, the filtering effect is not satisfying. Especially when filter length increases, the result is even worse than that of CA-CFAR. For this shortcoming, this paper improves WRF-CFAR and proposes cyclic weighed recursive filter algorithm. The diagram of CWRF -CFAR is shown in Fig.7. 


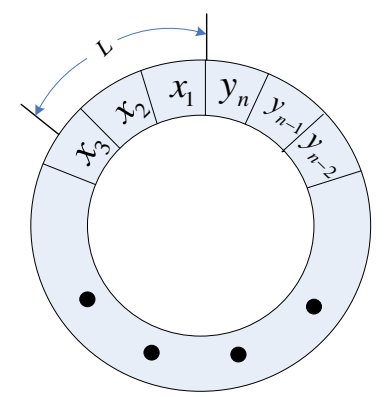

Fig.9 Loop filter sketch map

In uniform clutter background, sampled data after filtration and demodulation are connected head to end. Then the weighted recursive algorithm is adopted.

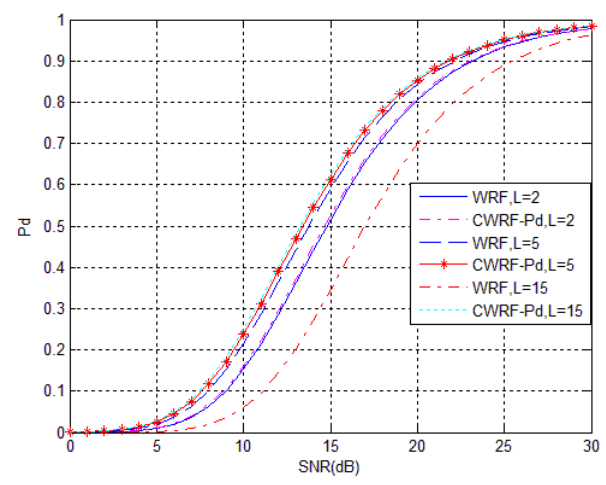

Fig.10 The compare of WRF-CFAR with CWRF-CFAR in homogeneous clutter background

Simulation results indicate that the improved CWRF-CFAR is superior to WRF-CFAR under different filtering length. The advantage is more obvious with the increase of $L$, for the detection effect of CWRF-CFAR keeps a slow enhancement while the detection effect of WRF-CFAR has a sharp decline.

\section{CONCLUSION}

In multiple targets environment, WRF-CFAR obtains recursive filtering threshold for filtering processing through processing sampled data in reference unit adaptively, thereby filtering out the influence of multiple targets in reference sliding window. Simulation results show that the detection performance of WRF-CFAR is better than CACFAR but worse than SO-CFAR in uniform clutter environment, while the false alarm control ability and comprehensive property of CA-CFAR is better than SO-CFAR. With the increase in the number of sliding windows, superiority of WRF-CFAR in detection performance and false control ability becomes more obvious when compared with CACFAR. The improved CWRF-CFAR has higher data utilization and have better filtering effects than WRF-CFAR.

\section{REFERENCES :}

C.Yi, Y.Yang, X.Z.Zhang. New CFAR target detector for SAR images based on kernel density estimation and mean square error distance[J]. IEEE Journal of Systems Engineering and Electronics.2012,23(1):40-46.

Dillard G M.Mean-level detection of nonfluctuating signals[J].IEEE Trans. On AES,1974,10(6):795-799.

Di Vito A,Moretti G. Probability of false alarm in CA-CFAR device downstream from linear-law detector[J]. Electronics Letters,1989,25(24):1692-1693.

Finn H M.A ,CFAR design for a window spanning two clutter fileds[J].IEEE Trans.on AES,1986,22(1):563-575.

Gandhi P P, Kassam S A. Analysis of CAFR processors in nonhomogeneous background[J].IEEE Trans .on AES, 1988,24(4):427-445.

GUI Ren-zhou.Using two-dimensional constant false alarm detection of nonuniform noise under the background of the target[J].Journal of wuhan university (information science edition) .2012,37(3):354-357. (in Chinese)

HAN Meng-fei,CUI Wei,WU Si-liang.A new radar fuse threshold frequency domain monitoring algorithm with constant false alarm rate[J].Acta Armamentarii. 2008,29(12):1422-1426. (in Chinese)

HE You,GUAN Jian,MENG Xiang-wei.Radar target detection and constant false alarm processing[M].Beijing:Tsinghua Univer Press,2011.

LIN Xu, HONG Jun, SUN Xian, YAN Yi. A model based on adaptive background clutter CFAR wide-swath SAR image ship detection algorithm[J].Remote Sensing Technology and Application. 2014,29(1):75-81. (in Chinese)

NI Fei, DENG Xing-cheng.Research on optical signal processing technology in space optical communication system[J].Laser Journal. 2000,21(4):10-12. (in Chinese)

Smith M E, Varshney P K. Intelligent CFAR processor based on data variability[J].IEEE Trans. on AES ,2000,36(3) :837847.

XU Yan-wei,HOU Zhao-huan,LI Jun,HAO Cheng-peng.The constant false alarm rate sampling particle filter technology and its application research[J].Applied Acoustics. 2013,32(4):320-324. (in Chinese)

YUAN Chang, YANG Li-chun, YAN Wei, YANG Wen. AN improved SAR images of ship detection algorithm with constant false alarm rate[J].Modern Radar. 2012,34(8): 29-32. (in Chinese) 